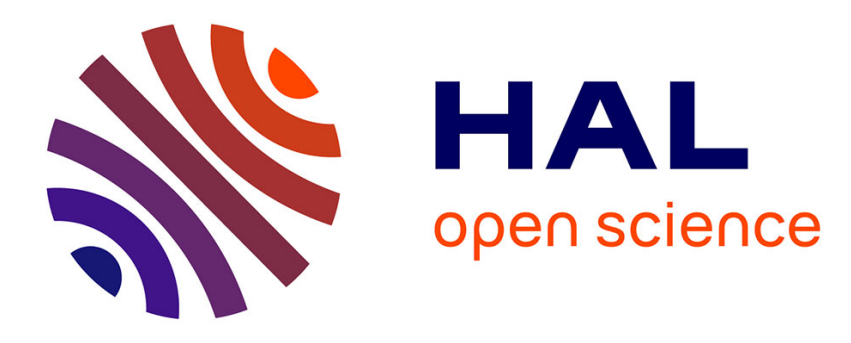

\title{
Latent heat identification in a two-phase Stefan problem
}

Muriel Gapaillard

\section{To cite this version:}

Muriel Gapaillard. Latent heat identification in a two-phase Stefan problem. IFAC World Congress, 2011, Milan, Italy. pp.10.3182/20110828-6-IT-1002.01393. hal-00626406

\section{HAL Id: hal-00626406 https://hal.science/hal-00626406}

Submitted on 26 Sep 2011

HAL is a multi-disciplinary open access archive for the deposit and dissemination of scientific research documents, whether they are published or not. The documents may come from teaching and research institutions in France or abroad, or from public or private research centers.
L'archive ouverte pluridisciplinaire HAL, est destinée au dépôt et à la diffusion de documents scientifiques de niveau recherche, publiés ou non, émanant des établissements d'enseignement et de recherche français ou étrangers, des laboratoires publics ou privés. 


\title{
Latent heat identification in a two-phase Stefan problem
}

\author{
Muriel Gapaillard \\ IRCCyN - UMR CNRS 6597, 1 rue de la Noé \\ BP 92101, 44321 Nantes Cedex 3, France \\ (e-mail: muriel.gapaillard@irccyn.ec-nantes.fr)
}

\begin{abstract}
A two-phase Stefan problem modelling a fusion or a solidification phenomenon is presented. It involves partial differential equations with discontinuities due to the latent heat. A regularisation of these equations is introduced, removing the discontinuities. Convergence results prove that it is possible to replace a problem of identifying the latent heat by a continuous problem issued from the regularisation. The difficulties inherent to the discontinuities are then avoided. A resolution method, based on the minimisation of a cost function by means of the calculus of variations is then presented.
\end{abstract}

\section{INTRODUCTION}

Hybrid systems admit a continuous dynamic in which discrete controlled or autonomous events occur [Van Der Schaft and Schumacher, 2000]. The modelling of the continuous process can be done by means of differential equations - it is the case when studying the behaviour of a thermostat [Quémard et al., 2005] or the movement of a car [D'Apice et al., 2003] - or by means of partial differential equations (PDEs) such as, for instance, in the study of the vibrations of a flexible beam [Castro and Zuazua, 1998]. The considered system is here a hybrid system with an autonomous model jump in which the continuous dynamic is described by means of PDEs. It is a two-phase Stefan problem (see for example Visintin [2008]), modelling a fusion or solidification process, that consists in determining the respective temperatures of the solid and liquid phases and also the position of the melting front.

Many studies have been devoted to the Stefan problem, in which theoretical [Friedman, 1968], numerical [Ciavaldini, 1975] or inverse [Slota, 2008] points of view have been emphasized. The study of such problems is made difficult by the discontinuities occurring in the state equation that accounts for the jump of the enthalpy. In order to avoid the use of specific methods such as variational inequalities, taking directly into account the discontinuous aspect of the problem, regularisation techniques have been developed to obtain continuity results with respect to the data [Niezgodka and Pawlow, 1983] or to obtain standard numerical algorithms [Grossmann et al., 2001]. The general principle of this approach, already used in the domain of hybrid optimal control [Bengea and DeCarlo, 2005, Gapaillard, 2008], is to prove convergence results allowing the replacement of discontinuous problems by regularised continuous systems, which are easier to solve.

We try here to identify the latent heat in a two-phase Stefan problem. The identification, computed from measurements, is set as the minimisation of a cost function. A resolution method using a regularisation of the problem is presented. It is based on convergence results allowing the replacement of the discontinuous PDEs of the original problem by continuous PDEs. The convergence of the solution of the regularised problem towards the solution of the original discontinuous problem is proved in an adequate functional context. The resolution of this new problem gives then an approximation of the latent heat, avoiding the difficulties due to the discontinuities. Indeed, the formalism of the calculus of variations is then applied in a classical and straightforward way to the regularised problem. The calculation of the gradient of the cost function is then greatly facilitated.

The original Stefan problem is presented in section 2 . Classical and enthalpy formulations are given as well as proof of the existence and uniqueness of the solution. The regularisation of the enthalpy function is introduced in section 3 , and the convergence of the regularised solution towards the solution of the initial problem is proved. The identification problem of the latent heat is presented in section 4 , in both the initial and regularised cases. The convergence between the two cases is proved. Finally, the detailed calculation of the gradient of the cost function is given for the regularised problem.

\section{TWO-PHASE STEFAN PROBLEM}

\subsection{Classical formulation}

We consider between instants 0 and $t_{\mathrm{f}}$, a melting/solidification problem where the liquid and solid phases belong to interval $\Omega=\left[0, L_{x}\right]$ of $\mathbb{R}$. We study temperature $\theta$ on domain $Q=\Omega \times\left[0, t_{\mathrm{f}}\right]$.

For each instant $t \in\left[0, t_{\mathrm{f}}\right]$ we set:

$$
\Omega=\Omega_{l}(t) \cup \Omega_{s}(t) \cup\left\{x_{\mathrm{f}}(t)\right\},
$$

where $\Omega_{l}(t)$ and $\Omega_{s}(t)$ correspond to liquid and solid phases respectively, and where $x_{\mathrm{f}}(t)$ represents the abscissa of the melting or solidification point (see Fig. 1).

By denoting:

$$
Q_{l}=\left\{(x, t) \in Q \mid x \in \Omega_{l}(t)\right\}
$$

and 


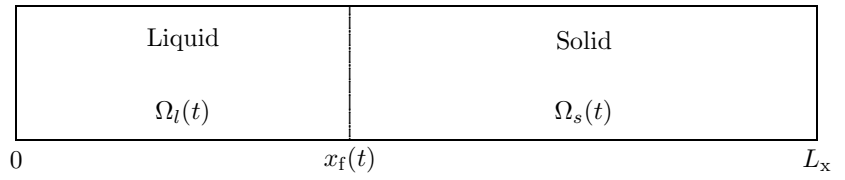

Fig. 1. Liquid and solid phases at instant $t$

$$
Q_{s}=\left\{(x, t) \in Q \mid x \in \Omega_{s}(t)\right\},
$$

we get the following equations:

$$
\begin{aligned}
\forall(x, t) \in & Q_{l} \\
& \rho_{1} C_{1} \frac{\partial \theta}{\partial t}(x, t)=\frac{\partial}{\partial x}\left(\lambda_{1} \frac{\partial \theta}{\partial x}\right)(x, t)+f(x, t) \\
\forall(x, t) \in & Q_{s} \\
& \rho_{2} C_{2} \frac{\partial \theta}{\partial t}(x, t)=\frac{\partial}{\partial x}\left(\lambda_{2} \frac{\partial \theta}{\partial x}\right)(x, t)+f(x, t)
\end{aligned}
$$

where $f \in L^{2}(Q)$ represents an external heat source and $\rho_{i}, C_{i}$ and $\lambda_{i}$ respectively denote the densities, the specific heat capacities and the heat conductivities in the liquid, for $i=1$, and solid phases, for $i=2$.

At the liquid/solid interface $\mathcal{S}$, defined by:

$$
\mathcal{S}=\left\{(x, t) \in Q \mid x=x_{\mathrm{f}}(t)\right\}
$$

we have :

$$
\forall(x, t) \in \mathcal{S} \quad \theta(x, t)=0
$$

and

$$
\begin{aligned}
& \forall(x, t) \in \mathcal{S} \\
& \qquad \rho_{2} L \frac{d x_{\mathrm{f}}}{d t}(t)=-\lambda_{1} \frac{\partial \theta}{\partial x}\left(x^{-}, t\right)+\lambda_{2} \frac{\partial \theta}{\partial x}\left(x^{+}, t\right),
\end{aligned}
$$

where $L$ corresponds to the latent heat.

The Fourier boundary conditions are given by:

$$
\begin{aligned}
\forall t \in\left[0, t_{\mathrm{f}}\right] & \lambda_{1} \frac{\partial \theta_{1}}{\partial x}(0, t)=\alpha_{0}\left(\theta_{1}(0, t)-\theta_{\operatorname{ex} 0}\right) \\
\forall t \in\left[0, t_{\mathrm{f}}\right] \quad \lambda_{2} \frac{\partial \theta_{2}}{\partial x}\left(L_{x}, t\right) & =-\alpha_{L_{x}}\left(\theta_{2}\left(L_{x}, t\right)-\theta_{\operatorname{ex} L_{x}}\right)
\end{aligned}
$$

where $\alpha_{0}$ and $\alpha_{L_{x}}$ are positive constants and $\theta_{\operatorname{ex} 0}$ and $\theta_{\operatorname{ex} L_{x}}$ represent the outside temperature at abscissae 0 and $L_{x}$ respectively.

As initial condition we have:

$$
\forall x \in \Omega \quad \theta(x, 0)=\theta_{0}(x)
$$

where $\theta_{0} \in L^{2}(\Omega)$ verifies:

$$
\theta_{0}(x) \neq 0 \quad \text { a.e. } x \in \Omega
$$

\subsection{Enthalpy formulation}

From equations (1) and (2), we get the following formulation:

$$
\begin{aligned}
& \forall(x, t) \in Q \backslash \mathcal{S} \\
& \quad C(\theta) \frac{\partial \theta}{\partial t}(x, t)=\frac{\partial}{\partial x}\left(\lambda(\theta) \frac{\partial \theta}{\partial x}\right)(x, t)+f(x, t)
\end{aligned}
$$

in which we set:

$$
\begin{array}{ll}
C(\theta)=\rho_{1} C_{1} & \text { if } \theta>0 \\
C(\theta)=\rho_{2} C_{2} & \text { if } \theta<0
\end{array}
$$

and

$$
\begin{array}{ll}
\lambda(\theta)=\lambda_{1} & \text { if } \theta>0 \\
\lambda(\theta)=\lambda_{2} & \text { if } \theta<0 .
\end{array}
$$

Let $\beta$ be the function defined by:

$$
\forall r \in \mathbb{R} \quad \beta(r)=\int_{0}^{r} \lambda(\tau) d \tau
$$

and let $\widetilde{H}$ be an enthalpy function defined over $\mathbb{R}$ by:

$$
\begin{aligned}
& \widetilde{H}(\theta)=\rho_{1} C_{1} \theta+\rho_{2} L \quad \text { if } \theta \geq 0 \\
& \widetilde{H}(\theta)=\rho_{2} C_{2} \theta \quad \text { if } \theta<0,
\end{aligned}
$$

where the temperature $\theta$ is in degrees Celsius except for an additive constant.

We then consider the following change in variable:

$$
v=\beta(\theta)
$$

and we set:

$$
H=\widetilde{H} \circ \beta^{-1} .
$$

Function $H$ is thus defined by:

$$
\begin{aligned}
& H(v)=\frac{\rho_{1} C_{1}}{\lambda_{1}} v+\rho_{2} L \quad \text { if } v \geq 0 \\
& H(v)=\frac{\rho_{2} C_{2}}{\lambda_{2}} v \quad \text { if } v<0 .
\end{aligned}
$$

Furthermore, remarking that:

$$
\forall(x, t) \in \mathcal{S} \quad v(x, t)=0,
$$

and that $\mathcal{S}$ is not necessarily a set of measure zero, the discontinuity of $H$ at 0 leads us to consider function $H(v)$ as a multivalued function.

We shall thus introduce a function $w$ such that:

$$
w \in H(v),
$$

which means:

$$
\begin{aligned}
& w(x, t)=H(v(x, t)) \quad \text { if }(x, t) \in Q \backslash \mathcal{S} \\
& w(x, t) \in\left[H\left(0^{-}\right), H(0)\right] \quad \text { if }(x, t) \in \mathcal{S} .
\end{aligned}
$$

With the help of the new variables $v$ and $w$, we obtain the enthalpy formulation of the Stefan problem introduced in the previous section. This formulation is given by the following equations:

$$
\begin{aligned}
& \forall(x, t) \in Q \backslash \mathcal{S} \quad \frac{\partial w}{\partial t}(x, t)=\Delta v(x, t)+f(x, t) \\
& \forall t \in\left[0, t_{\mathrm{f}}\right] \quad \frac{\partial v}{\partial x}(0, t)=g_{0}(v(0, t)) \\
& \forall t \in\left[0, t_{\mathrm{f}}\right] \quad \frac{\partial v}{\partial x}\left(L_{x}, t\right)=g_{L_{x}}\left(v\left(L_{x}, t\right)\right) \\
& \forall\left(x_{\mathrm{f}}(t), t\right) \in \mathcal{S} \quad v\left(x_{\mathrm{f}}(t), t\right)=0 \\
& \forall\left(x_{\mathrm{f}}(t), t\right) \in \mathcal{S} \\
& \rho_{2} L \frac{d x_{\mathrm{f}}}{d t}(t)=-\frac{\partial v}{\partial x}\left(x_{\mathrm{f}}(t), t\right)+\frac{\partial v}{\partial x}\left(x_{\mathrm{f}}(t), t\right)
\end{aligned}
$$

$\forall x \in \Omega \quad v(x, 0)=v_{0}(x)$

where we set:

$$
v_{0}=\beta\left(\theta_{0}\right)
$$

and where $g_{0}$ and $g_{L_{x}}$ are functions defined by:

$$
\forall v \in \mathbb{R} \quad g_{0}(v)=\alpha_{0}\left(\frac{v}{\lambda_{1}}-\theta_{\mathrm{ex} 0}\right)
$$

and 


$$
\forall v \in \mathbb{R} \quad g_{L_{x}}(v)=-\alpha_{L_{x}}\left(\frac{v}{\lambda_{2}}-\theta_{\operatorname{ex} L_{x}}\right) .
$$

Moreover, from (3), we have:

$$
v_{0}(x) \neq 0 \quad \text { a.e. } x \in \Omega \text {. }
$$

\subsection{Existence and uniqueness result}

In order to formulate existence and uniqueness result, we shall introduce the variational formulation associated with equations (4-9).

Let $\varphi \in H^{1}(Q)$ such that $\varphi\left(., t_{\mathrm{f}}\right)=0$ over $\Omega$.

By formally multiplying (4) by $\varphi$ and integrating over $Q$, we obtain:

$$
\left(\frac{\partial w}{\partial t}, \varphi\right)_{L^{2}(Q)}=(\Delta v, \varphi)_{L^{2}(Q)}+(f, \varphi)_{L^{2}(Q)} .
$$

By integrating by parts and using (10), we deduce the following equation:

$$
\begin{aligned}
& -\int_{\Omega} H\left(v_{0}(x)\right) \varphi(x, 0) d x-\left(w, \frac{\partial \varphi}{\partial t}\right)_{L^{2}(Q)} \\
= & \int_{0}^{t_{\mathrm{f}}} g_{L_{x}}\left(v\left(L_{x}, t\right)\right) \varphi\left(L_{x}, t\right) d t-\int_{0}^{t_{\mathrm{f}}} g_{0}(v(0, t)) \varphi(0, t) d t \\
& -\left(\frac{\partial v}{\partial x}, \frac{\partial \varphi}{\partial x}\right)_{L^{2}(Q)}+(f, \varphi)_{L^{2}(Q)}
\end{aligned}
$$

which defines the variational formulation of the two-phase Stefan problem.

We can now introduce, as in Nochetto [1985], the following definition:

Definition 1. A function $v \in L^{2}\left(0, t_{\mathrm{f}} ; H^{1}(\Omega)\right)$ is called weak solution of the Stefan problem given by (4-9) if there exists a function $w \in L^{2}(Q)$ such that:

- $w \in H(v)$;

- equation (11) holds for all functions $\varphi \in H^{1}(Q)$ with $\varphi\left(., t_{\mathrm{f}}\right)=0$ over $\Omega$.

Let us now give an existence, uniqueness and regularity result for the two-phase Stefan problem (see Niezgodka and Pawlow [1983] and Nochetto [1985] and its bibliography for the proof).

Theorem 2. We suppose that $\theta_{0} \in H^{1}(\Omega) \cap L^{\infty}(\Omega)$ and $f \in L^{\infty}(Q)$.

Then, there exists a unique weak solution $v$ in sense of Definition 1.

Moreover, we have:

$$
v \in L^{\infty}(Q) \cap L^{\infty}\left(0, t_{\mathrm{f}} ; H^{1}(\Omega)\right) \cap H^{1}\left(0, t_{\mathrm{f}} ; L^{2}(\Omega)\right) .
$$

\section{REGULARISATION OF THE STEFAN PROBLEM}

\subsection{Regularisation of $H$}

In order to obtain a regularisation of the Stefan problem introduced in section 2, it was decided to approach the function $H$ through a sequence of regular functions $\left(H_{n}\right)_{n>0}$ verifying the following conditions:

- for all $n>0, H_{n}$ belongs to class $\mathcal{C}^{2}$ over $\mathbb{R}$ and has first and second derivatives, $H_{n}^{\prime}$ and $H_{n}^{\prime \prime}$, bounded over $\mathbb{R}$;
- the sequence converges uniformly to $H$ on all compact sets of $\mathbb{R}$ that do no include 0 ;

- for all $n>0, H_{n}$ coincides with $H$ over the set:

$$
]-\infty, 0\left[\cup \left[\frac{1}{n},+\infty[\text {. }\right.\right.
$$

These properties will be required to prove the existence and uniqueness result in $\S 3.2$ as well as the convergence and continuity theorems in $\S \S 3.3$ and 4.2 .

From now on, we shall consider the sequence of functions $\left(H_{n}\right)_{n>0}$ defined over $\mathbb{R}$ by:

$$
\begin{aligned}
& H_{n}(v)=\frac{\rho_{1} C_{1}}{\lambda_{1}} v+\rho_{2} L \quad \text { if } v>\frac{1}{n} \\
& H_{n}(v)=a v^{5}+b v^{4}+c v^{3}+\frac{\rho_{2} C_{2}}{\lambda_{2}} v \quad \text { if } v \in\left[0, \frac{1}{n}\right] \\
& H_{n}(v)=\frac{\rho_{2} C_{2}}{\lambda_{2}} v \quad \text { if } v<0
\end{aligned}
$$

where we set:

$$
\begin{aligned}
a & =3\left(\frac{\rho_{1} C_{1}}{\lambda_{1}}-\frac{\rho_{2} C_{2}}{\lambda_{2}}\right) n^{4}+6 \rho_{2} L n^{5} \\
b & =-8\left(\frac{\rho_{1} C_{1}}{\lambda_{1}}-\frac{\rho_{2} C_{2}}{\lambda_{2}}\right) n^{3}-15 \rho_{2} L n^{4} \\
c & =6\left(\frac{\rho_{1} C_{1}}{\lambda_{1}}-\frac{\rho_{2} C_{2}}{\lambda_{2}}\right) n^{2}+10 \rho_{2} L n^{3} .
\end{aligned}
$$

\subsection{Regularised problem}

We introduce a regularised problem by replacing, in the enthalpy formulation (4-9), equation (4) by:

$$
\forall(x, t) \in Q \quad \frac{\partial H_{n}(v)}{\partial t}(x, t)=\Delta v(x, t)+f(x, t)
$$

and the initial condition (9) by:

$$
\forall x \in \Omega \quad v_{n}(x, 0)=v_{0, n}(x)
$$

where we set $v_{0, n}=H_{n}^{-1}\left(H\left(v_{0}\right)\right)$.

Boundary conditions are kept unchanged.

By multiplying equation (15) by a function $\varphi \in H^{1}(\Omega)$ and by integrating over $\Omega$, we obtain the variational formulation of the regularised problem. We can then introduce the following definition:

Definition 3. Let $n>0$.

A function $v_{n} \in L^{2}\left(0, t_{\mathrm{f}} ; H^{1}(\Omega)\right)$ is called a weak solution of the regularised problem if it verifies:

$$
\begin{aligned}
& \forall t \in\left[0, t_{\mathrm{f}}\right] \forall \varphi \in H^{1}(\Omega) \\
& \begin{array}{r}
\left(\frac{\partial H_{n}\left(v_{n}(., t)\right)}{\partial t}, \varphi\right)_{L^{2}(\Omega)}+\left(\frac{\partial v_{n}}{\partial x}(., t), \frac{\mathrm{d} \varphi}{\mathrm{d} x}\right)_{L^{2}(\Omega)} \\
+g_{0}\left(v_{n}(0, t)\right) \varphi(0)-g_{L_{x}}\left(v_{n}\left(L_{x}, t\right)\right) \varphi\left(L_{x}\right) \\
=(f(., t), \varphi)_{L^{2}(\Omega)}
\end{array}
\end{aligned}
$$

with the initial condition:

$$
\forall x \in \Omega \quad v_{n}(x, 0)=v_{0, n}(x)
$$

In the same way as for the initial Stefan problem, we give below an existence and uniqueness result for the regularised problem. 
Theorem 4. For all $n>0$, there exists, in sense of Definition 3 , a unique weak solution $v_{n}$.

Proof. The regularity hypotheses made on functions $H_{n}$ allow to use a proof developed in Burger and Pogu [1991]. It is sufficient to adapt this proof in order to take into account the boundary condition at $L_{x}$, which is assumed to be zero in Burger and Pogu [1991].

\subsection{Connection with the initial problem}

The following theorem ensures the convergence of the solution of the regularised problem to that of the initial problem.

Theorem 5. Let $v$ be the solution of the initial problem given by Theorem 2 .

For all strictly positive integer $n$, we note:

$$
A_{n}(v)=\left\{(x, t) \in Q,|v(x, t)| \leq \frac{1}{n}\right\}
$$

and we assume:

$$
\exists M \in \mathbb{R} \quad \forall n>0 \quad\left|A_{n}(v)\right| \leq M \frac{1}{n} .
$$

Then, sequence $\left(v_{n}\right)_{n>0}$, given by Theorem 4 , converges to $v$ in $L^{2}(Q)$ when $n$ tends to infinity.

Proof. This proof uses ideas developed in Grossmann et al. [2001] and Nochetto [1985] where Stefan problems are studied within other frameworks. Actually, Grossmann et al. [2001] consider Dirichlet boundary conditions and Nochetto [1985] studies an initial problem that does not include a function $w$ such that:

$$
w \in H(v) \text {. }
$$

Due to shortage of space, the following calculations will not be completely detailed.

Let $n>0$ and $\varphi$ the following test function:

$$
\forall(x, t) \in Q \quad \varphi(x, t)=\int_{t}^{t_{\mathrm{f}}} v_{n}(x, \tau)-v(x, \tau) d \tau .
$$

By using this function in (16) and by integrating over $\left[0, t_{\mathrm{f}}\right]$, we obtain by means of an integration by parts:

$$
\begin{aligned}
& -\int_{\Omega} H_{n}\left(v_{0, n}(x)\right) \varphi(x, 0) d x-\left(H_{n}\left(v_{n}\right), \frac{\partial \varphi}{\partial t}\right)_{L^{2}(Q)} \\
& +\left(\frac{\partial v_{n}}{\partial x}, \frac{\partial \varphi}{\partial x}\right)_{L^{2}(Q)}+\int_{0}^{t_{\mathrm{f}}} g_{0}\left(v_{n}(0, t)\right) \varphi(0, t) d t \\
& -\int_{0}^{t_{\mathrm{f}}} g_{L_{x}}\left(v_{n}\left(L_{x}, t\right)\right) \varphi\left(L_{x}, t\right) d t=(f, \varphi)_{L^{2}(Q)} .
\end{aligned}
$$

Equations (11) and (19) then allow to write:

$$
\begin{aligned}
&\left(H_{n}\left(v_{n}\right)-\right.\left.w, \frac{\partial \varphi}{\partial t}\right)_{L^{2}(Q)}=\left(\frac{\partial v_{n}}{\partial x}-\frac{\partial v}{\partial x}, \frac{\partial \varphi}{\partial x}\right)_{L^{2}(Q)} \\
&+\int_{0}^{t_{\mathrm{f}}}\left(g_{0}\left(v_{n}(0, t)\right)-g_{0}(v(0, t))\right) \varphi(0, t) d t \\
&-\int_{0}^{t_{\mathrm{f}}}\left(g_{L_{x}}\left(v_{n}\left(L_{x}, t\right)\right)-g_{L_{x}}\left(v\left(L_{x}, t\right)\right)\right) \varphi\left(L_{x}, t\right) d t .(20)
\end{aligned}
$$

With similar calculation techniques as used in Grossmann et al. [2001], we show that each term of the second member of equation (20) is positive. It then follows:

$$
\left(H_{n}\left(v_{n}\right)-w, \frac{\partial \varphi}{\partial t}\right)_{L^{2}(Q)} \geq 0 .
$$

Now, we have

$$
\left(H_{n}\left(v_{n}\right)-w, \frac{\partial \varphi}{\partial t}\right)_{L^{2}(Q)}=-\left(H_{n}\left(v_{n}\right)-w, v_{n}-v\right)_{L^{2}(Q)} .
$$

Therefore, by introducing $H_{n}(v)$, we therefore get:

$-\left(H_{n}(v)-w, v_{n}-v\right)_{L^{2}(Q)} \geq\left(H_{n}\left(v_{n}\right)-H_{n}(v), v_{n}-v\right)_{L^{2}(Q)}$.

By remarking that:

$$
\forall x \in \mathbb{R} \quad H^{\prime}{ }_{n}(x) \geq p,
$$

where we set $p=\min \left(\frac{\rho_{1} C_{1}}{\lambda_{1}}, \frac{\rho_{2} C_{2}}{\lambda_{2}}\right)$, the mean value inequality then allows to write:

$$
-\left(H_{n}(v)-w, v_{n}-v\right)_{L^{2}(Q)} \geq p\left\|v_{n}-v\right\|_{L^{2}(Q)}^{2} .
$$

Let us suppose $n>1$.

By using Cauchy-Schwarz inequality and properties of $A_{n}(v)$ and $H_{n}$ and by writing $Q$ as follows:

$$
Q=A_{n}(v) \cup Q \backslash A_{n}(v)
$$

we obtain:

$$
\left|\int_{Q}\left(H_{n}(v)-w\right)\left(v_{n}-v\right) d x d t\right| \leq K \frac{1}{\sqrt{n}}\left\|v_{n}-v\right\|_{L^{2}(Q)}
$$

where we set $K=\left(\rho_{2} L+\frac{\rho_{1} C_{1}}{\lambda_{1}}\right) \sqrt{M}$.

We thus deduce from (21):

$$
\left\|v_{n}-v\right\|_{L^{2}(Q)} \leq \frac{K}{p} \frac{1}{\sqrt{n}}
$$

which completes the proof.

Thanks to Theorem 5, it has been shown how the regularised problem allows to approach the initial Stefan problem. This result will be used in the next section in order to simplify the resolution of an identification problem.

\section{LATENT HEAT IDENTIFICATION}

\subsection{Identification problem and its regularisation}

The studied problem consists in identifying, from measurements, the latent heat $L$.

We set $\Omega_{L}=\left[L_{1}, L_{2}\right]$, where $L_{1}$ and $L_{2}$ are strictly positive real numbers. For $L \in \Omega_{L}$, we write $v(L ; .,$.$) the solution$ of the Stefan problem given by Theorem 2 .

The identification problem then consists in minimising on $\Omega_{L}$ the cost function defined by:

$\forall L \in \Omega_{L} \quad J(L)=\frac{1}{2} \int_{0}^{t_{\mathrm{f}}} \sum_{i=1}^{s}\left(v\left(L ; x_{i}, t\right)-\beta(\hat{\theta})\left(x_{i}, t\right)\right)^{2} d t$

where $s$ denotes the number of sensors and where $\hat{\theta}\left(x_{i}, t\right)$ is the measure of the temperature performed at the point of abscissa $x_{i}$ at instant $t$.

In order to establish the convergence result of $\S 4.2$, we consider a theoretical framework in which we have measurements at each point in time and space. In numerical applications, it will be sufficient to perform an interpolation from measures $\hat{\theta}\left(x_{i}, t\right)$. 
We then replace the above cost function by a new function, still denoted $J$, involving an observed state $\hat{v}$ defined over the whole domain $Q$. This function is defined by:

$$
\forall L \in \Omega_{L} \quad J(L)=\frac{1}{2}\|v(L ; ., .)-\hat{v}(., .)\|_{L^{2}(Q)}^{2} .
$$

The regularised formulation of this identification problem is defined with the help of the regularised Stefan problem introduced in section 3 .

For $n>0$, this new problem consists in minimising over $\Omega_{L}$ the cost function $J_{n}$ defined by:

$$
\forall L \in \Omega_{L} \quad J_{n}(L)=\frac{1}{2}\left\|v_{n}(L ; ., .)-\hat{v}(., .)\right\|_{L^{2}(Q)}^{2}
$$

where $v_{n}(L ; .,$.$) denotes the solution given by Theorem 4$.

\subsection{Convergence between solutions}

We shall show in Theorem 9 that the minimisation of $J_{n}$ allows to obtain an approximation of the minimum of $J$. This convergence result is based on the continuity of cost functions $J$ and $J_{n}$. Due to shortage of space, we only give the main arguments to prove these continuity results.

Theorem 6 . Let $n \geq 1$. Assume:

$$
f \in L^{\infty}(Q)
$$

and

$$
\theta_{0} \in H^{1}(\Omega) \cup L^{\infty}(Q)
$$

Then, the solutions $v$ of the initial Stefan problem and $v_{n}$ of the regularised problem are continuous with respect to $L$.

Moreover, $v$ and $v_{n}$ are uniformly bounded with respect to $L$.

Proof. The continuity of $v$ with respect to $L$ is obtained by means of changes of variables and by using a continuity result, with respect to the initial data, which is proved in Niezgodka and Pawlow [1983].

The continuity of $v_{n}$ is proved with the help of the continuity of $H_{n}^{\prime}$ with respect to $L$ and by using a continuity result, with respect to parameters, for a nonlinear parabolic system [Burger and Chen, 1995].

By studying the variations of $H_{n}^{\prime}$, we show that $H_{n}^{\prime}$ is bounded independently of $L$. A theorem, proved in Burger and Pogu [1991], then allows to obtain that $v_{n}$ is uniformly bounded with respect to $L$.

Finally, by using this result and the convergence of $v_{n}$ to $v$, we obtain that $v$ is uniformly bounded with respect to $L$.

Corollary 7. Functions $J$ and $J_{n}$ are continuous on interval $\Omega_{L}=\left[L_{1}, L_{2}\right]$.

Proof. Thanks to Theorem 6, we can use the Lebesgue dominated convergence theorem and achieve the announced result.

According to the above corollary, functions $J$ and $J_{n}$ reach their minimum values on the compact set $\Omega_{L}$ at points denoted $L^{\star}$ and $L_{n}^{\star}$ respectively. Now, since the sequence $\left(L_{n}^{\star}\right)_{n>0}$ is bounded, there exists a subsequence, which we do not relabel, that converges towards a point denoted $\bar{L}$.
In order to establish the convergence results of Proposition 8 and Theorem 9 below, we suppose that the constant $M$, given in Theorem 5 , is independent of the latent heat. Proposition 8. Sequence $\left(v_{n}\left(L_{n}^{\star} ; ., .\right)\right)_{n>0}$ converges to $v(\bar{L} ; .,$.$) in L^{2}(Q)$ when $n$ tends to inifinity.

Proof. We adapt the proof of Theorem 5 to the present case.

We replace $v, v_{n}, H$ and $H_{n}$ by $v(\bar{L} ; .,),. v_{n}\left(L_{n}^{\star} ; .,.\right), H(\bar{L})$ and $H_{n}\left(L_{n}^{\star}\right)$ respectively.

The proof is similar to that of Theorem 5 up to equation (21). For the continuation of the proof, the same mathematical tools, combined with the definitions of $H(\bar{L})$ and $H_{n}\left(L_{n}^{\star}\right)$, lead to:

$$
\begin{aligned}
& \left|\int_{Q}\left(H_{n}\left(L_{n}^{\star} ; v(\bar{L})\right)-w\right)\left(v_{n}\left(L_{n}^{\star}\right)-v(\bar{L})\right) d x d t\right| \\
& \quad \leq\left(\left(\rho_{2} L_{2}+\frac{\rho_{1} C_{1}}{\lambda_{1}}\right) \sqrt{M} \frac{1}{\sqrt{n}}+\rho_{2}\left|L_{n}^{\star}-\bar{L}\right| \sqrt{L_{x} t_{\mathrm{f}}}\right) \\
& \left\|v_{n}\left(L_{n}^{\star}\right)-v(\bar{L})\right\|_{L^{2}(Q)} .
\end{aligned}
$$

We complete then the proof by using the following inequality:

$$
\begin{array}{r}
\left\|v_{n}\left(L_{n}^{\star}\right)-v(\bar{L})\right\|_{L^{2}(Q)} \leq \frac{1}{p}\left(\left(\rho_{2} L_{2}+\frac{\rho_{1} C_{1}}{\lambda_{1}}\right) \sqrt{M} \frac{1}{\sqrt{n}}\right. \\
\left.+\rho_{2}\left|L_{n}^{\star}-\bar{L}\right| \sqrt{L_{x} t_{\mathrm{f}}}\right)
\end{array}
$$

remarking that the sequence $\left(L_{n}^{\star}\right)_{n>0}$ converges to $\bar{L}$ when $n$ tends to infinity.

Theorem 9. Sequence $\left(J_{n}\left(L_{n}^{\star}\right)\right)_{n>0}$ converges to $J\left(L^{\star}\right)$ when $n$ tends to infinity.

Proof. Let $n>0$.

The definition of $L_{n}^{\star}$ allows to write:

$$
\forall L \in \Omega_{L} \quad J_{n}\left(L_{n}^{\star}\right) \leq J_{n}(L) .
$$

Theorem 5 leads to:

$$
\forall L \in \Omega_{L} \quad \lim _{n \rightarrow+\infty} J_{n}(L)=J(L)
$$

and, according to Proposition 8, we have:

$$
\lim _{n \rightarrow+\infty} J_{n}\left(L_{n}^{\star}\right)=J(\bar{L}) .
$$

Equation (22) implies therefore:

$$
\forall L \in \Omega_{L} \quad J(\bar{L}) \leq J(L) .
$$

We deduce therefrom:

$$
J(\bar{L})=J\left(L^{\star}\right) .
$$

The claim is confirmed.

The above theorem shows that the sequence $\left(L_{n}^{\star}\right)_{n>0}$, obtained by minimising the functions $J_{n}$, converges to a point which achieves the minimum of $J$. Thus, if $L^{\star}$ is supposed to be unique and if we choose $n$ big enough,we can identify the latent heat by solving the regularised problem.

\subsection{Resolution method}

The resolution method introduced in this section consists in determining the gradient of the cost function $J_{n}$ with respect to the latent heat $L$. This expression is actually 
needed to implement descent methods in order to minimise $J_{n}$.

Difficulties associated with discontinuities can be avoided through the use of the regularised problem, justified by Theorem 9. It is therefore possible to obtain the gradient by means of classical techniques of the calculus of variations, as will now be shown.

We introduce an adjoint state $p_{n}$, where $p_{n} \in\left\{p:\left[0, t_{\mathrm{f}}\right] \rightarrow\right.$ $\left.H^{1}(\Omega)\right\}$, and a lagrangian as follows:

$$
\begin{gathered}
\mathscr{L}\left(v_{n}, p_{n} ; L\right)=J_{n}(L)+\left(\frac{\partial H_{n}\left(v_{n}\right)}{\partial t}, p_{n}\right)_{L^{2}(Q)} \\
+\left(\frac{\partial v_{n}}{\partial x}, \frac{\partial p_{n}}{\partial x}\right)_{L^{2}(Q)}+\int_{0}^{t_{\mathrm{f}}} g_{0}\left(v_{n}(0, t)\right) p_{n}(0, t) d t \\
-\int_{0}^{t_{\mathrm{f}}} g_{L_{x}}\left(v_{n}\left(L_{x}, t\right)\right) p_{n}\left(L_{x}, t\right) d t-\left(f, p_{n}\right)_{L^{2}(Q)}
\end{gathered}
$$

where, to alleviate the writing, we used $v$ and $v_{n}$ instead of $v(L ; .,$.$) and v_{n}(L ; .,$.$) respectively.$

After integrations by parts and by using the initial condition (17), we obtain:

$$
\begin{gathered}
\mathscr{L}\left(v_{n}, p_{n} ; L\right)=J_{n}(L)-\left(H_{n}\left(v_{n}\right), \frac{\partial p_{n}}{\partial t}\right)_{L^{2}(Q)} \\
-\left(v_{n}, \frac{\partial^{2} p_{n}}{\partial x^{2}}\right)_{L^{2}(Q)}-\left(f, p_{n}\right)_{L^{2}(Q)} \\
+\int_{0}^{t_{\mathrm{f}}}\left(g_{0}\left(v_{n}(0, t)\right) p_{n}(0, t)-v_{n}(0, t) \frac{\partial p_{n}}{\partial x}(0, t)\right) d t \\
-\int_{0}^{t_{\mathrm{f}}}\left(g_{L_{x}}\left(v_{n}\left(L_{x}, t\right)\right) p_{n}\left(L_{x}, t\right)-v_{n}\left(L_{x}, t\right) \frac{\partial p_{n}}{\partial x}\left(L_{x}, t\right)\right) d t \\
+\int_{\Omega}\left(H_{n}\left(v_{n}\left(x, t_{\mathrm{f}}\right)\right) p_{n}\left(x, t_{\mathrm{f}}\right)-H_{n}\left(v_{0, n}(x)\right) p_{n}(x, 0)\right) d x .
\end{gathered}
$$

By choosing $p_{n}$ to cause the Gâteaux-derivative of $\mathscr{L}$ with respect to $v_{n}$ to vanish, we obtain the following adjoint system:

$$
\begin{aligned}
& \forall(x, t) \in Q \quad H^{\prime}{ }_{n}\left(v_{n}(x, t)\right) \frac{\partial p_{n}}{\partial t}(x, t)=-\frac{\partial^{2} p_{n}}{\partial x^{2}}(x, t) \\
& \quad+v_{n}(x, t)-\hat{v}(x, t)
\end{aligned} \quad \begin{aligned}
& \forall t \in\left[0, t_{\mathrm{f}}\right] \quad \frac{\partial p_{n}}{\partial x}(0, t)=\frac{\alpha_{0}}{\lambda_{1}} p_{n}(0, t) \\
& \forall t \in\left[0, t_{\mathrm{f}}\right] \quad \frac{\partial p_{n}}{\partial x}\left(L_{x}, t\right)=-\frac{\alpha_{L_{x}}}{\lambda_{2}} p_{n}\left(L_{x}, t\right) \\
& \forall x \in \Omega \quad p_{n}\left(x, t_{\mathrm{f}}\right)=0 .
\end{aligned}
$$

We notice that the function $H_{n}$, defined by $(12-14)$, is differentiable with respect to $L$. This derivative will be denoted $\frac{\partial H_{n}}{\partial L}$.

The lagrangian derivation with respect to $L$ then gives the following expression of the gradient of the cost function:

$$
\begin{aligned}
\nabla\left(J_{n}\right)_{L}=-\left(\frac{\partial H_{n}}{\partial L}\left(v_{n}\right)\right. & \left., \frac{\partial p_{n}}{\partial t}\right)_{L^{2}(Q)} \\
& -\rho_{2} \int_{\left\{x \in \Omega, v_{0}(x)>0\right\}} p_{n}(x, 0) d x
\end{aligned}
$$

where $p_{n}$ is the solution of (23-26) and where we used the initial condition (17).

\section{CONCLUSION}

An identification method of the latent heat in a two-phase Stefan problem has been presented. It is based on the regularisation of the jump occurring in the enthalpy formulation of the Stefan problem. Convergence results have allowed to replace the initial discontinuous problem by a continuous one. This approach leads to a straightforward use of the calculus of variations.

In a future work, this study may be extended to the twodimensional case or to other problems such as optimal control problems.

\section{REFERENCES}

S. Bengea and R. A. DeCarlo. Optimal control of switching systems. Automatica, 41(1), 11-27, 2005.

J. Burger and M. Chen. Optimal input for the identification of the parameters of a nonlinear parabolic system. Systems Analysis Modelling Simulation, 20(4), 243-254, 1995.

J. Burger and M. Pogu. Functional and Numerical Solution of a Control Problem Originating from Heat Transfer. Journal of Optimization Theory and Applications, 68(1), 49-73, 1991.

C. Castro and E. Zuazua. Boundary controllability of a hybrid system consisting in two flexible beams connected by a point mass. SIAM Journal on Control and Optimization, 36(5), 1576-1595, 1998.

J. F. Ciavaldini. Analyse numérique d'un problème de Stefan à deux phases par une méthode d'éléments finis. SIAM Journal on Numerical Analysis, 12(3), 464-487, 1975.

C. D'Apice, M. Garavello, R. Manzo and B. Piccoli. Hybrid optimal control: case study of a car with gears. International Journal of Control, 76(13), 1272-1284, 2003.

A. Friedman. The Stefan problem in several space variables. Transactions of the American Mathematical Society, 133, 51-87, 1968.

M. Gapaillard. Continuous representation and control of hybrid systems. International Journal of Control, 81(1), 1-20, 2008.

C. Grossmann, H. P. Scheffler and S. Winzer. Smoothing and Newton's method for a discontinuous variational equation of a Stefan type. Optimization, 49(1), 115-136, 2001.

R. H. Nochetto. Error estimates for two-phase Stefan problems in several space variables, II: Non-linear flux conditions. Calcolo, 22(4), 501-534, 1985.

M. Niezgodka and I. Pawlow. A Generalized Stefan Problem in Several Space Variables. Applied Mathematics and Optimization, 9, 193-224, 1983.

C. Quémard, J. C. Jolly and J. L. Ferrier. Mathematical study of a thermal device as a hybrid system. Proceedings of IMACS World Congress, 2005.

D. Slota. Using genetic algorithms for the determination of an heat transfer coefficient in three-phase inverse Stefan problem. International Communications in Heat and Mass Transfer, 35(2), 149-156, 2008.

A. Van Der Schaft and H. Schumacher. An Introduction to Hybrid Dynamical Systems. Springer-Verlag, London, 2000.

A. Visintin. Introduction to Stefan-type problems. In C. Dafermos and M. Pokorny, editors, Handbook of Differential Equations: Evolutionary Equations, volume 4, pages 377-484. North-Holland, Amsterdam, 2008. 